Helgoländer wiss. Meeresunters. 17, 381-391 (1968)

\title{
Deichsicherung mit Verhüttungsrückständen
}

\author{
Manfred Haucke \\ Hoesch AG Hüttenwerke, Dortmund
}

\begin{abstract}
Dike proctection by metallurgic residues. Residues and scrap heaps as well as mud and dust of the smelting industry can be used for protecting dikes along the East-Frisian coast. A pretest (1340 $t$ of residues of the Hoesch Concern) and a test $(100,000 \mathrm{t}$ ) have been made along the East-Frisian west coast from 1964 to 1966. This suggested procedure helps the industry to get rid of residues and metallurgic wastes and protects dikes by creating a larger forefield. Getting rid of waste products was up to now a serious handicap for the metallurgic industry; there was not sufficient space available in the vicinity of the foundries; the resulting dumps and mud ponds make precious living districts rather worthless; there are legal impediments in the freshwater areas against the dumping of metallurgic residues. The composition and chemical analysis of the residues, the biological consequences of the new procedure suggested, and its usefulness in dike constructions are discussed.
\end{abstract}

\section{EINLEITUNG}

Dieser Beitrag behandelt die Unterbringung von Rückständen aus der Eisen- und Stahlindustrie im Küstenvorfeld Ostfrieslands, wobei es gelang, die Sorgen der Industrie bezüglich Abfallbeseitigung sinnvoll den Interessen des aktiven Küstenschutzes nutzbar zu machen.

Die Anstrengungen der Industrie zur Reinhaltung von Luft und Wasser im Binnenlande haben mit zum Teil großem finanziellem Aufwand beachtliche Erfolge aufzuweisen. Demgegenüber ist die Beseitigung beziehungsweise Unterbringung fester Industrieabfälle (inklusive Stäube und Schlämme) technisch und wirtschaftlich noch nicht befriedigend gelöst.

Die Gründe der Hoesch AG Hüttenwerke, mit den nicht in die metallurgischen Prozesse repetierfähigen und unverkäuflichen Reststoffen - im folgenden mit „Verhüttungsrückstände“ bezeichnet - an die Küste zu gehen, sind: (a) Verknappung des Kipp-Volumens in Hüttennähe, das heißt im industriellen Ballungsgebiet; (b) Wasserhaushaltsgesetze (von Bund und Ländern) schränken die Deponie-Möglichkeiten im Trinkwassereinzugs- (Süßwasser-) Gebiet immer mehr ein; besonders spürbar ist das bei Genehmigungsverfahren für neue Schuttkippen; (c) Kippen, Halden und Schlammteiche machen wertvolles Industrie- und Wohngelände meist unbrauchbar; (d) wirtschaftliche Gesichtspunkte.

Ursprünglich bestand die Absicht, an der Küste im Salz- und Brackwassergebiet lediglich langfristig nutzbare, für Hüttenreststoffe geeignete Ablagerungsflächen zu 
suchen. In Zusammenarbeit mit den für den Küstenschutz zuständigen Behörden ergab sich die Idee, mit Hilfe dieser Massen regelrechte Vorländer zur Verstärkung und Sicherung der Deiche aufzubauen.

\section{ERGEBNISSE UND DISKUSSION}

\section{Durchgeführte Versuche}

Bei allen Versuchen wurden Verhüttungsrückstände vor dem schar liegenden Seedeich am Campener Leuchtturm vorgeschüttet und einplaniert. Uber die geographischen Verhältnisse gibt Abbildung 1 Auskunft. Das Versuchsfeld liegt an der ostfriesischen Westküste, am Ostufer der Emsmündung.

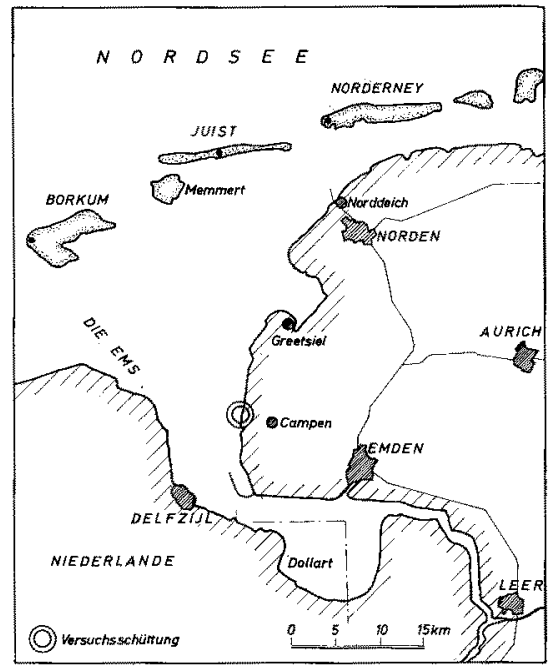

Abb. 1: Lage der Vorschüttung vor dem Seedeich bei Campen (Ostfriesland)

In einem orientierenden Vorversuch (1964) wurden $1340 \mathrm{t}$ in Form eines kleinen Dreieckprofils vor das Deichdeckwerk angeschüttet. Die Ergebnisse dieses Versuches ermutigten zu einem Großversuch (1965) mit 100000 t Verhüttungsrüdkständen in Form eines sich $100 \mathrm{~m}$ seewärts erstreckenden Profils (Abb. 2, oberes Bild). Schließlich wurde versucht, die vordere Kante der Schüttung mit 2000 t Material (1966) zu verbessern.

\section{Herkunft und Zusammensetzung der Verhüttungsrückstände}

Der weit überwiegende Anteil der Nebenprodukte und Reststoffe eines gemischten Hüttenwerkes werden aufbereitet und dann entweder verkauft oder in die metallurgischen Prozesse zurückgeführt. Trotzdem hinterbleiben 50 bis $100 \mathrm{~kg} / \mathrm{t}$ erzeugtem 
Rohstahl Verhüttungsrückstände, die beseitigt beziehungsweise untergebracht werden müssen.

Bei den Versuchen wurde ein möglichst repräsentativer Querschnitt aus diesen Stoffen eingesetzt. Garantiert ausgenommen waren Ole, Fette, radioaktives Material und Müll. Die Herkunft des Materials läßt sich wie folgt kennzeichnen: Feuerfestes Material aus Ofen- und Pfannenausbrüchen: Dolomit, Schamotte, Magnesit, Silikat u. a.; Rest-Schlacken aus der Roheisen- und Stahlerzeugung; Gießerei-Rückstände aus Abstichrinnen und Gießgruben; Schlämme aus der Gasreinigung des Hochofens (Gichtgasreinigungsschlamm) und aus der Naßreinigung anderer Betriebe; Neutralisationsschlamm aus Beizbetrieben; Stäube aus Entstaubungsanlagen, Dachstaub etc.; schließlich der bei Bauvorhaben anfallende Bauschutt und Erdaushub.

Alle festen Stoffe (außer Schlamm) werden einer Aufbereitungsanlage zugeführt, in der sie vom Eisen befreit und durch Siebung klassiert werden. Bei langfristig fast gleicher Zusammensetzung des Zulaufs entstehen durch den Aufbereitungsprozeß ziemlich homogene Komponenten, deren chemische Analyse und Kornverteilung sich nur wenig ändert. Dies betrifft insbesondere die für das folgende sehr wichtige Hauptkomponente Feinschutt 0 bis $8 \mathrm{~mm}$.

Die im Großversuch 1965 eingebauten 100000 t Material verteilen sich auf verschiedene Komponenten (vgl. Tabelle 1).

Tabelle 1

Zusammensetzung der Verhüttungsrückstände

\begin{tabular}{|lcc|}
\hline Bezeichnung & Korngröße & Gewichtsprozente \\
\hline (1) Feinschutt & $0-8 \mathrm{~mm}$ & $32,5 \%$ \\
(2) Grobschutt & $8-120 \mathrm{~mm}$ & $16,0 \%$ \\
(3) Geröll (= „Katzenköpfe“) & $120-400 \mathrm{~mm}$ & $6,5 \%$ \\
(4) Gießerei-Schutt (= Sand) & $0-3 \mathrm{~mm}$ & $9,5 \%$ \\
(5) Gichtgasreinigungsschlamm & $0-1 \mathrm{~mm}$ & $3,5 \%$ \\
(6) Erdaushub & nicht bestimmt & $7,5 \%$ \\
(7) Mischgut (aus 1 bis 5) & $0-500 \mathrm{~mm}$ & $24,5 \%$ \\
\hline
\end{tabular}

Chemische Analyse und Meerwasserlöslichkeit

Die chemische Analyse der Hauptkomponenten ist in Tabelle 2 wiedergegeben. Die gröberen Anteile des Grobschutts und ebenso das Geröll haben natürlich vom Feinschutt abweichende Stückanalysen. Es handelt sich ganz überwiegend um feuerfeste Steine und Schlackenbrocken, deren Einzelanalysen in summa der angegebenen Feinschuttanalyse entsprechen. Bei der Stoffart 7 handelt es sich um Material, das für den Großversuch von der Schuttkippe wieder aufgenommen wurde und dessen $\mathrm{Zu}$ sammensetzung der prozentualen Verteilung der Komponenten 1 bis 5 entspricht.

Bemerkenswert beim Feinschutt sind die hohen Werte für $\mathrm{CaO}=15,4 \%$ und $\mathrm{SiO}_{2}=47,66 \%$. Das Material hat also fast so etwas wie Zement-Charakter, und man kann sich daraus seine abbindenden und verfestigenden Eigenschaften erklären. Dies gilt analog auch für Grobschutt. Der Gießereischutt ist im wesentlichen ein durch 
384

M. Haucke

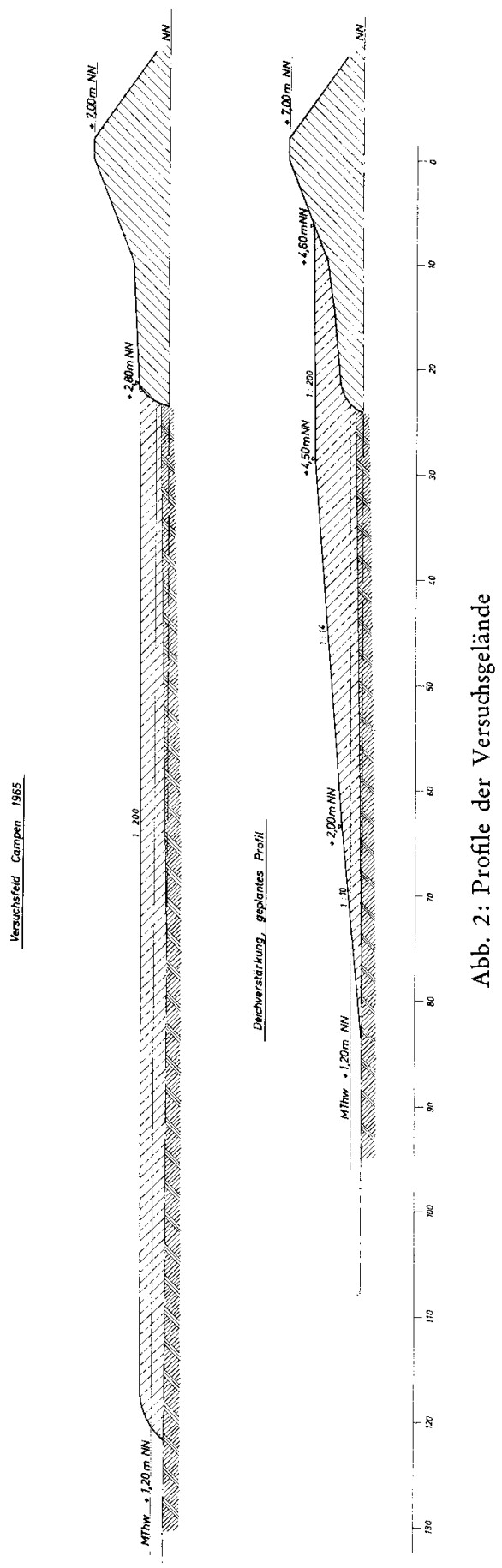


Tabelle 2

Chemische Analyse der Verhüttungsrückstände

\begin{tabular}{|c|c|c|c|}
\hline Komponenten & in- und Grobschutt & Gießereischutt & $\begin{array}{c}\text { Gichtgas- } \\
\text { Reinigungsschlamm }\end{array}$ \\
\hline $\mathrm{Fe}$ & 10,64 & 4,82 & 23,94 \\
\hline berechnet als $\mathrm{Fe}_{2} \mathrm{O}_{3}$ & 15,20 & 6,90 & 34,20 \\
\hline $\mathrm{MnO}$ & 2,28 & 0,23 & 1,14 \\
\hline $\mathrm{P}_{2} \mathrm{O}_{5}$ & 0,85 & 0,26 & 1,33 \\
\hline $\mathrm{SiO}_{2}$ & 47,66 & 77,80 & 11,40 \\
\hline $\mathrm{Al}_{2} \mathrm{O}_{3}+\mathrm{TiO}_{2}$ & 7,61 & - & 7,65 \\
\hline $\mathrm{CaO}$ & 15,40 & 4,40 & 10,00 \\
\hline $\mathrm{MgO}$ & 5,83 & 1,29 & 3,16 \\
\hline $\mathrm{Cr}_{2} \mathrm{O}_{3}$ & 0,08 & - & - \\
\hline Ges. S & 0,27 & - & 0,27 \\
\hline Sulfat & 0,81 & - & - \\
\hline Ges. C & 3,49 & 0,77 & 9,61 \\
\hline $\mathrm{CO}_{2}$ & 0,42 & - & - \\
\hline $\mathrm{ZnO}$ & $<0,01$ & - & 8,96 \\
\hline $\mathrm{Na}_{2} \mathrm{O}$ & 0,35 & - & 0,12 \\
\hline $\mathrm{K}_{2} \mathrm{O}$ & 0,73 & - & 0,24 \\
\hline Glïhrückstand & 95,12 & 96,42 & \\
\hline Glühverlust & 4,88 & 3,58 & \\
\hline chemisch gebundenes $\mathrm{H}_{2} \mathrm{C}$ & 2,03 & 1,28 & 8,20 \\
\hline
\end{tabular}

Eisenoxid verunreinigter Sand. Der aus der Gasreinigung des Hochofens stammende Gichtgasreinigungsschlamm enthält über das chemisch gebundene Wasser von $8,2 \%$ hinaus trotz jahrelanger Ablagerung noch einen ungebundenen Wassergehalt von ca. $40 \%$. Im Feststoffanteil dieses tixotropen Schlammes befindet sich überwiegend $(34,2 \%$ ) Eisenoxid und ein bemerkenswert hoher Anteil Zinkoxid (ca. 9\%).

Tabelle 3

Auslaugungsversuch mit Nordseewasser nach DIN 50900.

Verwendung von künstlichem Meerwasser nach DIN 50900; Standversuch: 96 Stunden Einwirkungszeit; Mischungsverhältnis Probe und Wasser 1:1; Auslaugungswasser vor Untersuchung filtriert

\begin{tabular}{|llcccc|}
\hline Analysenwerte & & $\begin{array}{c}\text { Feinschutt } \\
0-8 \mathrm{~mm}\end{array}$ & Meerwasser & Zunahme & Abnahme \\
\hline pH-Wert & & 9,2 & 7,8 & 1,4 & \\
Leitfähigkeit & $\mathrm{mS} / \mathrm{cm}$ & 47,5 & 46,5 & 1,0 & \\
Gesamthärte & $0 \mathrm{dH}$ & 303 & 346 & & \\
Calcium & $\mathrm{g} / 1$ & 1,38 & 0,32 & 1,06 & 0,83 \\
Magnesium & $\mathrm{g} / 1$ & 0,48 & 1,31 & & \\
Natrium & $\mathrm{g} / 1$ & 11,90 & 11,30 & 0,60 & \\
Kalium & $\mathrm{g} / 1$ & 0,08 & 0,02 & 0,06 & \\
Sulfate & $\mathrm{g} / 1$ & 3,04 & 2,79 & 0,25 & \\
Chloride & $\mathrm{g} / 1$ & 19,99 & 19,70 & 0,29 & \\
Phenole & $\mathrm{g} / 1$ & 0,0002 & & & \\
Rhodanide & $\mathrm{g} / 1$ & n.n. & & & \\
Cyanide & $\mathrm{g} / 1$ & n.n. & & & \\
\hline
\end{tabular}


Die Löslichkeit der eingesetzten Stoffe wurde durch Auslaugungsversuche mit Nordsee-Wasser nach DIN 50900 belegt. Da bei den Versuchen zur Deichvorschüttung praktisch nur der Feinschutt $O$ bis $8 \mathrm{~mm}$ mit dem Meerwasser in Berührung kommt, sind in Tabelle 3 nur die Werte für dieses Material angegeben. Es ist ersichtlich, daß der Gesamt-Salzgehalt des Wassers nur geringfügig verändert wird. Bei der Auslaugung ist der pH-Wert gestiegen, die Leitfähigkeit hat sich leicht erhöht. Während der Gehalt an Calcium sich um das 4fache erhöht, verringert sich der Magnesiumgehalt erheblich. Diese Verringerung des Mg-Gehaltes wird verursacht durch die Erhöhung der Alkalität (Reaktion des Wassers mit Kalk) und der dann möglichen Bildung unlöslicher Magnesium-Verbindungen.

\section{Aufbau der Vorschüttung}

Das Versuchsfeld bei Campen erstreckt sich $200 \mathrm{~m}$ längs des Seedeichs und $100 \mathrm{~m}$ seewärts (vgl. Abb. 2 oben und Abb. 3, 4, 5).

Ein Streifen mit Grobschutt und Geröll wurde vor dem Fußdeckwerk des Deiches als Drainage und „Rippen“ aus dem gleichen Material senkrecht zur Deichlinie direkt auf den Wattboden eingebracht. Der Kern der Schüttung wird aus einer Mischung aller

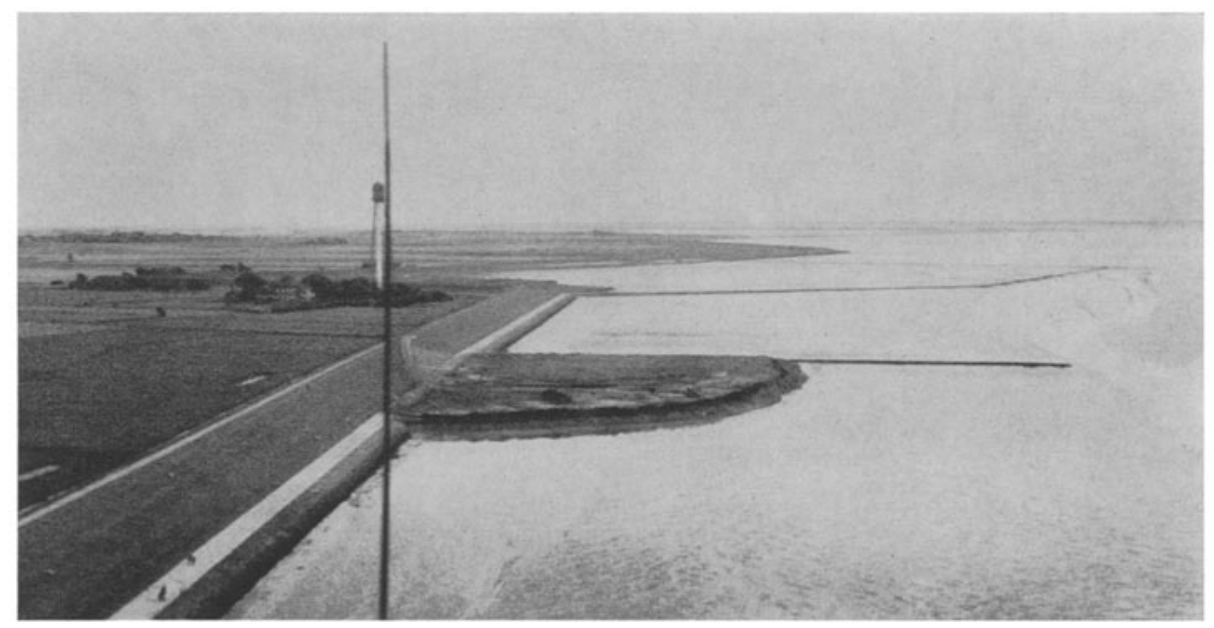

Abb. 3: Die Vorschüttung während der Baumaßnahme 1965 am Campener Leuchtturm. Blickrichtung nach Süden. Die Steinbuhne am südlichen Rand der Schüttung hat eine Länge von $175 \mathrm{~m}$. Der helle (Beton-)Streifen am Außendeich ist die Außenberme, davor das schwere Fußdeckwerk

oben genannten Komponenten gebildet. Die obere Abdeckung bildet ein sehr kalkreicher Feinschutt $O$ bis $8 \mathrm{~mm}$, der in einer Schichtdicke von 50 bis $70 \mathrm{~cm}$ genau in der Höhe des geplanten Profils einplaniert wird. Die Verhüttungsrückstände und besonders der Feinschutt haben verfestigende beziehungsweise verfrittende Eigenschaften, so daß mit dem Material ein regelrechtes Bauwerk erstellt werden kann. Die Herstellung 


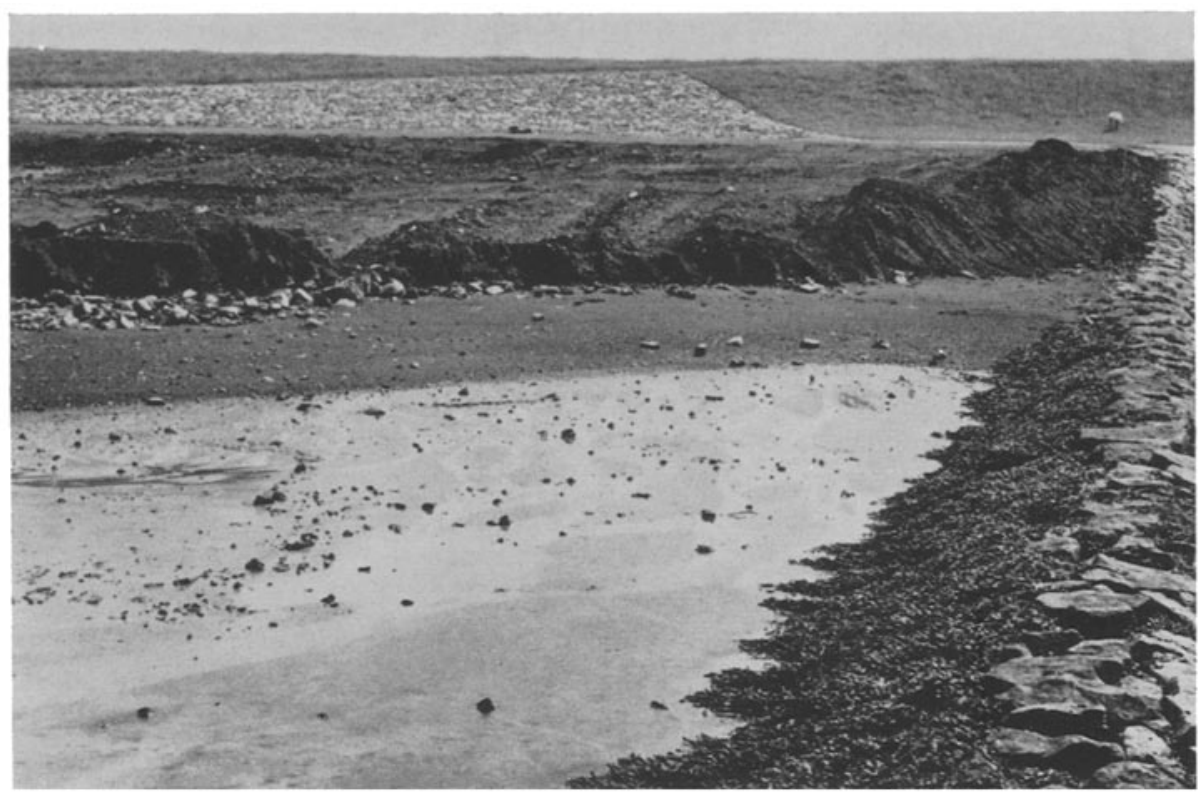

Abb. 4: Das Einbringen der Verhüttungsrückstände. Im Vordergrund Wattboden (hell), die dunklen Materialhaufen sind Feinschutt. Rechts die südlich begrenzende Steinbuhne. Im Hintergrund der Seedeich (Krone: $+7,00 \mathrm{~m} \mathrm{NN}$ ) mit befestigter Uberfahrt

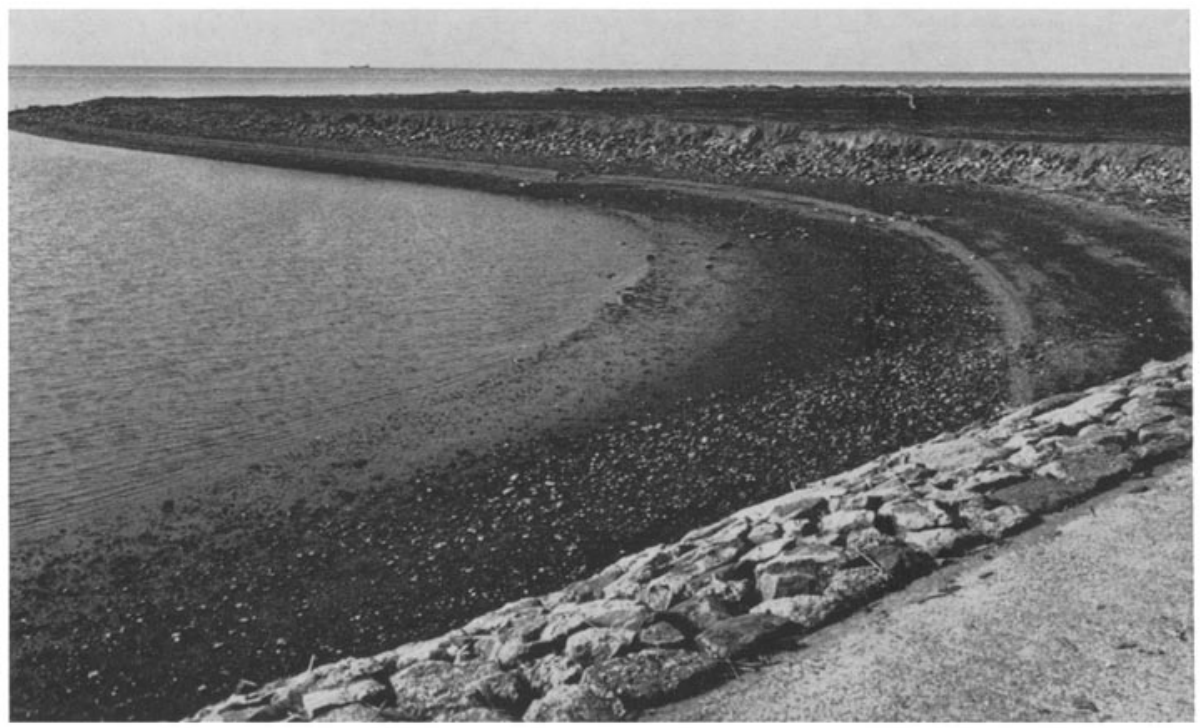

Abb. 5: Blick auf die südliche Begrenzung des Schüttkörpers. An der noch nicht abgeflachten Abbruchkante ist die Uberdeckung des groben Materials mit Feinschutt erkennbar. Vorn Außenberme und schweres Deichdeckwerk 
der Vorschüttung geschieht durch einfaches Abkippen und schichtweises Einplanieren. Das Schüttprofil lehnt sich im Süden an eine Steinbuhne (die sogenannte „Ohlingshöfe") an. An der Deich-Außenberme schließt es in einer Höhe von $2,80 \mathrm{~m}$ NN an und fällt geringfügig mit 1:200 seewärts ab. Die äußere Begrenzung wurde als steile Abbruchkante (1:3) belassen, deren Gefälle sich unter der Einwirkung des Angriffes der See - erwartungsgemäß - auf etwa 1:14 eingestellt hat.

\section{Auswirkungen für den Küstenschutz}

Hauptzweck der Deichvorschüttung mit Verhüittungsrückständen ist die Sicherung beziehungsweise Verstärkung des Seedeiches. Die Minderung des Wellenangriffs ist beachtlich und wird wie folgt belegt: Das Plateau der Vorschüttung hat eine Höhenlage von 1,60 m (am Deich) beziehungsweise 1,30 m (Vorderkante) über MThw (vgl. Abb.2 oben). Die Oberfläche der Vorschüttung wird nur bei sehr hohen Sturmfluten überspült. Die Wellen werden schon an der seeseitigen Böschung gebrochen, die Energie der Wellen wird auf dem langen Weg $(100 \mathrm{~m})$ zum Deich weitgehend verbraucht. Im Bereich der Versuchsschüttung wurde der Wellenauflauf bei zwei sehr hohen Sturmfluten, die den Schüttkörper ganz überströmten, um einen Meter gesenkt, was an der Lage der Treibselgrenze genau abgelesen werden kann.

Die Wirkung der hydraulischen Kräfte auf einen Schüttkörper nach Art des Großversuchs 1965 als auch auf ein aus den gewonnen Erfahrungen entwickeltes neues Profil (Abb. 2 unten) sind Gegenstand eingehender Erörterungen im „Küstenausschuß Nord- und Ostsee“ im Fachgebiet Küstenschutz. Das geplante Profil berücksichtigt von vornherein den sich natürlich einstellenden Böschungswinkel und ist am Deichkörper bis auf $+4,60 \mathrm{~m} \mathrm{NN}$ hochgezogen. Bei diesem wesentlich höheren Dreiecksprofil hofft man, jede andere Deichverstärkung und -erhöhung zu ersparen (gegebenenfalls sogar die landseitige Böschung zu belassen), das heißt den Deich dann überhaupt nicht mehr anzufassen.

Umfangreiche Untersuchungen zur Eignung des Materials in bodenphysikalischer Hinsicht wurden von der Forschungsstelle Norderney durchgeführt. Diese Untersuchungen schließen ein: die Erfassung aller Feststoffbewegungen auf dem Schïttkörper als auch im umgebenden Wattgebiet, insbesondere auch genaueste Nivellements der Profile und der seeseitigen Begrenzungen. Untersucht wurde die Lagestabilität, die Witterungsbeständigkeit, die Kohäsionseigenschaften, die Porösität und anderes mehr.

Im Brandungsbereich ist eine absolute Lagestabilität nicht erreichbar; das Material entmischt sich, die Feinanteile agglomieren und verfestigen sich insbesondere auf der Oberfläche der Feinschuttabdeckung sehr stark. Das gröbere Material wird ausschließlich in Richtung Deich auf den Schüttkörper transportiert und bildet dort einen Brandungswall. Ein Materialtransport in Richtung See, also in den vorgelagerten Wattboden findet nicht statt, ein Materialverlust konnte nicht festgestellt werden. Demzufolge kann auch ein Abwandern oder Verdriften der Verhüttungsrïckstände in fernere Gebiete, zum Beispiel zum Emsfahrwasser hin, eindeutig ausgeschlossen werden. 


\section{Biologische Konsequenzen}

Bei der Diskussion der biologischen Verhältnisse sind drei Bereiche zu unterscheiden: (a) die Wechselwirkung des eingesetzten Materials mit dem freien Meerwasser, (b) die Auswirkungen auf das umgebende Watt und (c) auf den Schüttkörper selbst beziehungsweise seine Oberfläche.

Die möglichen Veränderungen oder Schädigungen des Meerwassers können aus den Löslichkeiten der eingesetzten Stoffe hergeleitet werden (Tab. 3). Die Abdeckung besteht ausschließlich aus dem oben genannten Feinschutt $O$ bis $8 \mathrm{~mm}$, die Eindringtiefe des Wassers im Sinne einer Auslaugung ist gering. Von den biologisch interessierenden Löslichkeiten der Cyanide, Rhodanide und Phenole wurden nur Phenole mit $0,0002 \mathrm{~g} / 1$ nachgewiesen. Selbst wenn die gesamte Deckschicht mit $70 \mathrm{~cm}$ Dicke beziehungsweise 25000 t Material am Stoffaustausch beteiligt wären, würden maximal 5000 g gelöst werden können. An der Wechselwirkung mit dem Meerwasser nimmt tatsächlich nur der vordere Randstreifen von 10 bis $15 \mathrm{~m}$ Breite, und zwar mit der Frequenz und der Uberspüldauer der Flut teil. Der Vorgang des Ubergangs gelöster Stoffe in das Wasser der freien See dürfte also sehr langsam und sukzessive vonstatten gehen.

Zur Feststellung und Beweissicherung des gegenwärtigen Wattzustandes und zur späteren Abschätzung möglicher Einwirkungen auf den örtlich vorhandenen Biotop wurden biologisch-ökologische Untersuchungen vor Beginn des Versuches durch die Forschungsstelle Norderney ausgeführt. Mögliche Folgen der Aufschüttung auf die biologischen Verhältnisse des umliegenden Wattes werden durch analoge Vergleichsuntersuchung noch ausstehen.

In diesem Zusammenhang sei hingewiesen auf die außerordentlich genauen und ausführlichen Untersuchungen der Forschungsstelle durch MülLER (1959, 1965). Es wurde zwar festgestellt, daß der Wattbiotop bis an die Schüttung, also an die neue Vorlandkante heranreicht; ferner scheint sich zu zeigen, daß in der Nähe der Schüttung der Polychät Heteromastus überwiegt, was Verunreinigungen anzeigen kann - aber nicht anzeigen muß. Schon diese Aussagen werden durch die Forschungsstelle Norderney nur unter Vorbehalt gemacht werden, da die genaue Kontrolle und Vergleichsuntersuchung noch ausstehen.

Das für den Schüttkörper verwendete Material ist mit an Sicherheit grenzender Wahrscheinlichkeit steril. Eventuell siedelnde Mikroorganismen konnten bisher nicht festgestellt werden. Eine wesentliche Voraussetzung für den Erfolg der Aufschüttung muß unter anderem auch darin gesehen werden, ob es gelingt, die Oberfläche zu begrünen. Die Möglichkeiten hierzu sollen im Rahmen von Versuchen in naher Zukunft geklärt werden. Ohne Eingriffe bisher hat sich eine spärliche Ruderal-Flora herausgestellt. Unter Binnenverhältnissen ist es möglich, ohne aufwendige, stützende Maßnahmen die Schüttkörper zu begrünen. Ob die im Binnenlande siedelnden Pflanzen und Pflanzengemeinschaften auch auf den Vorschüttungen im Küstenbereich angesiedelt werden können, soll ebenfalls durch Versuche geklärt werden. Das Geröll der seeseitigen Kante ist auch durch Aufsitzer (Grünalgen und Blasentang) besiedelt.

Eine Untersuchung der Untersuchungs- und Forschungsanstalt der Landwirtschaftskammer Weser-Ems in Oldenburg im Hinblick auf pflanzenschädliche Stoffe weist das Material als landwirtschaftlich unbedenklich aus. Anläßlich der behördlichen 
Genehmigung für die Deichvorschüttung wurden auf Grund chemischer und bodenkundlicher Untersuchungen keine Bedenken gegen das Einbringen der Hüttenrückstände geltend gemacht. Leider kann zu diesem Zeitpunkt noch nichts Abschließendes oder Vollständiges zu den biologischen Folgen gesagt werden. Es wird aber nach $\mathrm{Ab}-$ schluß der Untersuchungen zu gegebener Zeit nachgeholt werden.

\section{ZUSAMMENFASSUNG}

1. Es wird gezeigt, wie sich bei dem Verfahren der Deichsicherung mit Verhüttungsrückständen die Interessen der Industrie - Beseitigung von Reststoffen - mit denen des Küstenschutzes - Verstärkung der Seedeiche - mit Vorteil für beide Seiten verbinden lassen.

2. Art, Herkunft, chemische Analysen und die Meerwasserlöslichkeit insbesondere des Feinschutts, mit dem der Schüttkörper abgedeckt ist, werden angegeben.

3. Die Bewährung der Deichvorschüttung zum Schutz des Seedeichs ist erwiesen; der Zusammenhang der Gestaltung des Schüttprofils mit der Wirkung der hydraulischen Kräfte wird behandelt.

4. Die biologischen Konsequenzen werden diskutiert. Die biologisch-ökologischen Untersuchungen des umgebenden Wattgebietes vor Beginn der Untersuchungen liegen vor, die analogen Vergleichsuntersuchungen zur Beweissicherung des biologischen Wattzustandes nach den durchgeführten Maßnahmen sind noch nicht abgeschlossen. Es ist zu hoffen, daß sich die ökologischen Bedingungen für den Biotop nicht nachteilig ändern.

5. Der Stofftransport in fester wie gelöster Form, sowohl in das umgebende Watt wie in die freie See, ist relativ zur eingebrachten Menge außerordentlich gering. Es besteht daher die Erwartung, daß mögliche negative Auswirkungen sich in engen Grenzen halten.

\section{ZITIERTE LITERATUR}

Mùller, C. D., 1959. Fauna und Sediment in der Leybucht. Biologisch-bodenkundliche Untersuchung ... Jber. ForscbSt. Norderney 11, 39-178.

- 1965. Das ostfriesische Watt von Neuharlingersiel bis Harlesiel. Biologisch-sedimentologische Untersuchung ... Jber. ForschSt. Norderney 17, 139-154.

\section{Diskussion im Anschluß an den Vortrag HAUCKE}

ManN: Hat man schon untersucht, ob biologisch wrirksame Stoffe ausgelaugt werden können? Ich denke dabei insbesondere an Phenole, Schwermetallsalze, Rhodanide, Cyanide etc., also Stoffe, die in den Verschüttungsrückständen enthalten sein können.

HaUckE: Uber die biologische Wirkung dieser Stoffe läßt sich jetzt noch nichts sagen, da die Ergebnisse der Vergleichsuntersuchungen abgewartet werden müssen. Diese sollen ebenso ausführlich und gründlich durchgeführt werden wie die biologisch-ökologische Beweissicherung vor dem Großversuch. 
HEYDEMANN: Ihre Untersuchungen praktischer ökologisch-experimenteller Art durch Großeingriffe in Lebensräume haben mich sehr interessiert. Bevor man über die Ergebnisse einer biologischen Verträglichkeit solcher Eingriffe publiziert bzw. verallgemeinert, miißte man noch einen anderen Typ des Vorlandes mit solchen Großaufschüttungen untersuchen, nämlich den des bewachsenen Vorlandes. Sie hatten uns in Ihren Bildern nur das unbewachsene Vorland gezeigt, aber wir wissen ja, daß die Fauna im bewachsenen und unbewachsenen außerordentlich verschieden ist. Insofern wïrde mich interessieren, inwieweit Sie bei der Verfolgung dieses Ziels auch in Areale hineinkommen, die faunistisch vielseitiger sind als die Areale, die bisher für Ihre Untersuchungen in Frage kamen.

Haucke: Wir können uns aus wirtschaftlichen Gründen die Gebiete nicht aussuchen, so daß wir uns auf absehbare Zeit mit diesem Watt-Typ beschäftigen werden. In Küstenabschnitten mit hochliegenden natürlichen Vorländern, z. B. den reich bewachsenen Hellergebieten, bedarf der Deich keines zusätzlichen Schutzes (also auch keiner Vorschüttung). Es ist klar, daß z. B. in Hellergebieten völlig andere biologische Konditionen vorherrschen. Aber auch hier würde gelten: innerhalb des Schüttkörpers ist praktisch kein Leben; die Ungebung wird durch lösliche Stoffe nur wenig beeinflußt.

Mrchaelis: Zur Frage Herrn Heydemanns möchte ich sagen, daß nur schar liegende Deiche durch diese Schüttungen geschützt werden sollen; schon vorhandenes Vorland soll im natürlichen Zustand erhalten bleiben. 